\title{
Uso de la gamificación para trabajar paradojas estadísticas y mejorar el espíritu crítico de los estudiantes
}

\author{
Daniel Palací-López ${ }^{a}$, Jesús Palacíb \\ ${ }^{a}$ Universitat Politècnica de València, dapalpe@gmail.com, ${ }^{\mathrm{b}}$ Universidad Pontificia de Comillas, \\ jpalaci@comillas.edu
}

\section{Resumen}

Una de las mayores dificultades encontradas por los estudiantes en asignaturas de tipo cuantitativo en diversas asignaturas a nivel universitario parece ser la asimilación y aplicación de conceptos matemáticos, así como el análisis crítico de los resultados obtenidos.

Con objeto de mejorar la adquisición de este tipo de competencias esenciales para los estudiantes (correcta definición de un problema planteado, identificación y aplicación de las herramientas matemáticas apropiadas para su resolución, y análisis crítico de los resultados), en este trabajo se presenta una propuesta de planificación de actividades para una sesión práctica. Dichas actividades se programan para tener lugar tanto dentro como fuera del aula, previas, durante, y posteriores a la sesión presencial.

A modo de actividad previa se plantea el visionado de material audiovisual que aborda dos paradojas estadísticas (de Simpson o de Yule-Simpson, y de Ellsberg) y el problema de Monty Hall, que servirán como preparación para las actividades posteriores y como preámbulo a una actividad de debate abierto dentro del aula (actividades de inicio y de desarrollo).

Además, en las actividades de desarrollo durante la sesión presencial, y haciendo uso de la gamificación, en grupo de 3 o 4 estudiantes y, con ayuda de dados y cartas, los estudiantes se familiarizan de forma directa con los conceptos vistos y discutidos anteriormente, cuyos resultados se presentarán y discutirán en un debate posterior que se hace extensivo a todo al aula.

Palabras clave: resultados de aprendizaje, paradojas estadísticas, innovación docente. 


\section{Introducción}

Es un hecho aceptado de forma casi generalizada que uno de los escollos con los que tanto el profesorado como el alumnado universitario se encuentran, en asignaturas de carácter cuantitativo, es la dificultad de asimilación y aplicación de conceptos matemáticos por parte del estudiantado. A esta dificultad, se suele añadir el problema derivado de una correcta interpretación de los resultados obtenidos. Una de las posibles consecuencias de este problema es el incremento de las tasas de abandono (Cabrera, 2015). En los últimos años, con la finalidad de solventar en la medida posible esta problemática se ha incorporado, al proceso de enseñanza-aprendizaje, el uso de Tecnologías de la Información y la Comunicación (TIC) así como de material multimedia que ha dado muy buenos resultados (Casasús, Ivars y López, 2018). También se ha incorporado, de manera más que efectiva, la denominada gamificación en el aula, esto es, el uso de actividades de tipo lúdico cuyo objetivo es optimizar el proceso de aprendizaje (Agudo Garzón, et al.2014; Authors, 2014). En esta línea, en el trabajo que se presenta, se proponen una serie de actividades a aplicar en una unidad didáctica correspondiente a una asignatura de corte cuantitativo (Estadística) del grado de Ingeniería Química, ofertado por la Universitat Politécnica de València (UPV). El objetivo de las mismas es paliar las deficiencias relacionadas con las competencias ligadas a la definición de los problemas planteados, así como la identificación y aplicación de las herramientas matemáticas apropiadas para su resolución, y el análisis crítico de los resultados. Las actividades propuestas deberían desarrollarse, de manera coordinada, antes, durante y después de la sesión presencial. Cabe indicar que la asignatura, correspondiente al primer curso de Ingeniería Química, consta de 4 unidades didácticas (U.1: Introducción, U.2.: Estadística Descriptiva, U.3: Probabilidad y U.4: Inferencia Estadística), le corresponde una carga lectiva de 6 ECTS y es impartida por el Departamento de Estadística e Investigación Operativa Aplicadas y Calidad (DEIOAC). Otras cuestiones de interés se corresponden con: a) El tamaño del grupo: es de entre 50 y 60 alumnos en las sesiones de teoría y de entre 18 y 24 alumnos en las sesiones de práctica y b) Las competencias y resultados del aprendizaje que, de acuerdo con la guía docente de la propia asignatura y para la titulación en la que se enmarca se encuentran en la Tabla 1. 
Tabla 1. Competencias y resultados de aprendizaje

- Competencia específica:
CE01 - Resolver problemas matemáticos que puedan plantearse en la ingeniería. Aplicar los
conocimientos sobre álgebra lineal, geometría, geometría diferencial, cálculo diferencial e
integral, ecuaciones diferenciales y en derivadas parciales, métodos numéricos, algorítmica
numérica, estadística y optimización.
Competencias generales:
CG28 - Desarrollar la creatividad.
CG27- Tomar decisiones y razonar de forma crítica.
CT01 - Comprensión e integración:
La adquisición de esta competencia tendría lugar mediante la resolución de preguntas
de comprensión integradas en los ejercicios a llevar a cabo durante el curso, y su
evaluación se efectuaría mediante preguntas de control y/o rúbricas.
LT03 - Análisis y resolución de problemas:
distintos problemas asociados a cada una de las unidades temáticas, y su evaluación se
efectuaría mediante la revisión de los problemas resueltos por los alumnos a través de
PoliformaT.

Fuente: Elaboración Propia

Tomando como base esta información y la propuesta de contenidos de Romero y Zúnica (2008), la planificación que se propone para la impartición de la asignatura, atendiendo a las 4 unidades didácticas, se encuentra en la Tabla 2, teniendo en cuenta que se distingue las actividades que se llevarán a cabo en dos tipos: ‘teóricas' y 'prácticas':

Tabla 2. Unidades didácticas: composición y tiempo estimado

\begin{tabular}{|l|l|c|c|}
\hline \multirow{2}{*}{ UNIDAD DIDÁCTICA } & \multirow{2}{*}{ TEMAS } & \multicolumn{2}{|c|}{ TIEMPO ESTIMADO } \\
\cline { 2 - 4 } & & Teoría & Práctica \\
\hline U.1. & Tema 0 & 0'5 horas & - \\
\hline U.2. & Temas 1 y 2 & 5 '5 horas & 5 horas \\
\hline U.3. & Temas 3 a 7 & 11 horas & 15 '5 horas \\
\hline U.4. & Temas 8 a 12 & 13 horas & 9'5 horas \\
\hline
\end{tabular}

Donde, la información acerca del contenido de los temas que las constituyen, así como la estimación del tiempo necesario para su impartición se recoge en la Tabla 3. 
Uso de la gamificación para trabajar paradojas estadísticas y mejorar el espíritu crítico de los estudiantes

Tabla 3. Desglose de contenidos y tiempos/unidades didácticas

\begin{tabular}{|c|c|c|c|}
\hline \multirow[t]{2}{*}{ U.D. } & \multirow{2}{*}{ TEMAS } & \multicolumn{2}{|c|}{ TIEMPO ESTIMADO } \\
\hline & & Teoría & Práctica \\
\hline U.1. & Tema 0: Introducción & 0'5 hora & 0 horas \\
\hline \multicolumn{2}{|r|}{ TOTAL } & 0'5 horas & 0 horas \\
\hline U.2. & $\begin{array}{l}\text { Tema 1: Estadística descriptiva unidimensional } \\
\text { Tema 2: Estadística descriptiva bidimensional }\end{array}$ & $\begin{array}{l}2 \text { horas } \\
3 \text { '5 horas }\end{array}$ & $\begin{array}{l}\text { 2'5 horas } \\
2,5 \text { horas }\end{array}$ \\
\hline \multicolumn{2}{|r|}{ TOTAL } & 5'5 horas & 5 horas \\
\hline U.3. & $\begin{array}{l}\text { Tema 3.- Conceptos básicos de cálculo de } \\
\text { probabilidades }\end{array}$ & 2'5 horas & 5 horas \\
\hline U.3. & $\begin{array}{l}\text { Tema 4.- Distribuciones de Probabilidad } \\
\text { Tema 5.- Modelos específicos univariantes discretos } \\
\text { Tema 6.- Modelos específicos univariantes continuos } \\
\text { Tema 7.- Modelos específicos bivariantes }\end{array}$ & $\begin{array}{l}2 \text { horas } \\
2 ' 5 \text { horas } \\
3 \text { horas } \\
1 \text { hora }\end{array}$ & $\begin{array}{l}\text { 3'5 horas } \\
\text { 3'5 horas } \\
\text { 3'5 horas } \\
0 \text { horas }\end{array}$ \\
\hline \multicolumn{2}{|r|}{ TOTAL } & 11 horas & 15'5 horas \\
\hline U.4. & $\begin{array}{l}\text { Tema 8.- Introducción a la Inferencia } \\
\text { Tema 9.- Estimación por intervalos } \\
\text { Tema 10.- Contrastación de hipótesis } \\
\text { Tema 11.- Análisis de la Varianza } \\
\text { Tema 12.- Modelos de regresión lineal }\end{array}$ & $\begin{array}{l}1 ' 5 \text { horas } \\
2 \text { horas } \\
\text { 3'5 horas } \\
\text { 3'5 horas } \\
\text { 2'5 horas }\end{array}$ & $\begin{array}{l}0 \text { horas } \\
\text { 1'75 horas } \\
\text { 3'25 horas } \\
3 \text { horas } \\
1 ' 5 \text { horas }\end{array}$ \\
\hline \multicolumn{2}{|r|}{ TOTAL } & 13 horas & 9'5 horas \\
\hline
\end{tabular}

Así, la sesión seleccionada para la planificación de las actividades es una sesión práctica de hora y media dedicada a aplicación y análisis crítico de los conceptos/teoremas correspondientes al tema 3 , sombreado en la Tabla 3 (se prevé que el número de alumnos con los que se trabaje sea de entre 18 y 24 alumnos). Se seleccionó este tema por considerarse el más adecuado para la implementación de actividades en las que se puede fomentar el espíritu crítico de los estudiantes, especialmente frente a la existencia de paradojas estadísticas que podrían llevar al equívoco si no se comprenden bien y a falta de buenas capacidades de análisis crítico. Esto es posible, además, a partir únicamente de los conocimientos adquiridos hasta este tema, sin añadir la complejidad (innecesaria para este objetivo) de temas posteriores.

Las competencias a trabajar en esta sesión serían las siguientes: a) Definir correctamente sucesos condicionados y no condicionados; b) Calcular correcta y eficientemente la probabilidad de que ocurra un suceso; c) Aplicar el teorema del producto y de Bayes y d) Analizar de forma crítica las implicaciones prácticas de las probabilidades de sucesos condicionados y sin condicionar (paradoja de Simpson o de Yule-Simpson), y de no conocer "cómo funcionan las probabilidades" (paradoja de Ellsberg + Problema de Monty 
Hall). Y para trabajar estas competencias se proponen una serie de actividades, dentro y fuera del aula, previas a la sesión presencial, durante ésta y tras la misma. Dichas actividades, aparecen detalladas en el siguiente epígrafe.

\section{Propuesta}

Como se ha indicado anteriormente, en el presente epígrafe se indican los 3 tipos de actividades (previa, de inicio y de desarrollo), así como los resultados de aprendizaje que se espera obtener, los recursos necesarios y el método de evaluación empleado en cada caso.

\subsection{Actividad previa}

En esta actividad se plantea el visionado de material audiovisual que aborda dos paradojas estadísticas (de Simpson o de Yule- Simpson, y de Ellsberg) y el problema de Monty Hall, que servirán como preparación para las actividades posteriores y como preámbulo a una actividad de debate abierto dentro del aula (actividades de inicio y de desarrollo). Los datos identificativos de dicha actividad (título, tiempo estimado necesario para su puesta en marcha, materiales, instrucciones, ...) se recogen en la Tabla 4.

Tabla 4. Diseño de la actividad previa

\begin{tabular}{|l|}
\hline \multicolumn{1}{|c|}{ ACTIVIDAD PREVIA } \\
\hline Nombre: 'El problema de Monty Hall: ¿quieres la cabra o el coche?' \\
\hline Duración estimada: 45 minutos \\
\hline Materiales (disponibles en el apartado 'Tareas' o ‘Exámenes' de PoliformaT): \\
- Problemas cortos correspondientes al Tema 3 (respuesta cerrada o numérica). \\
- Formulario de la asignatura. \\
- Enlace vídeo: https://www.youtube.com/watch?v=BzAhrFrnpGM \\
- Cuestionario con 2-3 preguntas sobre el vídeo (archivo .pdf/.doc/.docx). \\
\hline Instrucciones: \\
- Los estudiantes deben realizar los problemas cortos del Tema 3 en el apartado 'Exámenes' de \\
PoliformaT, sin tiempo límite y pudiendo usar el formulario de la asignatura. Una vez enviado, \\
puede ver los resultados (pero no las respuestas correctas, que puede consultar al profesor en el \\
aula si tiene dudas). Este ejercicio no pesa sobre la nota final de la asignatura, pero facilita el \\
seguimiento al profesor y la preparación de los alumnos para la sesión presencial. \\
- Seguidamente, deben visualizar el vídeo y explicar por qué "cambio de variable” no es la \\
expresión adecuada, y que justifiquen matemáticamente el resultado mostrado en el vídeo. La \\
resolución de este ejercicio se debe subir mediante la aplicación de PoliformaT en el apartado \\
'Tareas', y se valorará positivamente su correcta resolución en la nota final de la asignatura. \\
\hline
\end{tabular}

Fuente: Elaboración Propia 


\subsection{Actividad de inicio}

Con la finalidad de comprobar que han realizado correctamente la actividad previa, al comienzo de la clase, se realizará una actividad que permita tener un feedback más o menos inmediato del alumnado. La actividad consistirá en cumplimentar un cuestionario que versará sobre los dos ejes que constituyen la actividad previa (repaso de conceptos previos y visualización y comprensión del vídeo). La información detallada se recoge en la Tabla 5.

Tabla 5. Diseño de la actividad de inicio

\begin{tabular}{|l|}
\hline \multicolumn{1}{|c|}{ ACTIVIDAD DE INICIO } \\
\hline Nombre: 'Cuestionario $3 / 3$ - Probabilidad condicionada' \\
\hline Duración estimada: 15 minutos \\
\hline Materiales: \\
- Test "Problema de Monty Hall” en 'Exámenes' de PoliformaT. \\
- Formulario de la asignatura (impreso o en .pdf). \\
\hline Instrucciones: \\
Cada estudiante, o pareja según número de alumnos y ordenadores disponibles, debe responder el \\
test ubicado en el apartado 'Exámenes' de PoliformaT. El tiempo límite para responder al test es de \\
10 minutos. En caso de ir por parejas, ambos miembros de la pareja responden el mismo test: \\
primero uno, y luego el otro con su usuario. Con un tiempo límite total de 15 minutos. Las \\
preguntas son cuestiones con respuesta cerrada o numérica, y cada estudiante debe guardar y enviar \\
para calificar su cuestionario con su usuario personal. \\
Se recomienda avisar de antemano a los alumnos para que no se retrasen, y bloquear el acceso a los \\
tests (varios modelos) con usuario y contraseña que se les proporcionará al inicio de la sesión, y \\
que cambiará de una sesión a otra. \\
\hline Evaluación: \\
- La evaluación en este caso se lleva a cabo de la forma tradicional con tests de respuesta cerrada o \\
numérica, admitiendo un pequeño margen de error por posibles aproximaciones para las preguntas \\
de respuesta numérica. \\
- Los resultados y respuestas correctas de este cuestionario no se harán públicos hasta que todos los \\
grupos hayan completado esta sesión de aula (si hay más de un grupo). No obstante, el profesor \\
dispondrá de los resultados de forma inmediata para saber en qué cuestiones incidir más o menos \\
en la fase de desarrollo.
\end{tabular}

Fuente: Elaboración Propia

Los resultados de aprendizaje que se pretenden evaluar con esta actividad son: a) Aplicar el teorema del producto correctamente; b) Aplicar correctamente el teorema de Bayes; c) Calcular probabilidades de sucesos condicionados y/o sin condicionar.

\subsection{Actividad de desarrollo}

La finalidad de la actividad de desarrollo es la de profundizar en los aspectos más prácticos de los conceptos impartidos en la sesión teórica previa y su aplicación, y como 
continuación de lo visto por los alumnos en la actividad previa. En dicha actividad, que se llevaría a cabo durante la sesión presencial, y haciendo uso de la gamificación, los alumnos se organizarían en grupo de 3 o 4 estudiantes y, con ayuda de dados y cartas, se familiarizan de forma directa con los conceptos visualizados y discutidos anteriormente. Concretamente, $\mathrm{y}$ al igual en las actividades anteriores, una tabla (6) recoge de forma pormenorizada todo lo relativo a la actividad de desarrollo. Dado que la definición de gamificación y los criterios que permiten identificar qué constituye o no un ejemplo de gamificación son variados (Seaborn y Fels, 2015), conviene aclarar el uso en este caso del concepto de "gamificación" en el sentido del uso de juegos de azar conocidos, y en especial sus reglas y los fundamentos (estadísticos) en que se basan, como base para establecer estrategias de victoria (o minimización de la probabilidad de derrota), y para comprender cómo el "mal uso" de la estadística puede servir para ocultar trampas implementadas en los propios elementos de los juegos de azar (e.g. dados cargados y la paradoja de Yule-Simpson).

Tabla 6. Diseño de la actividad de desarrollo

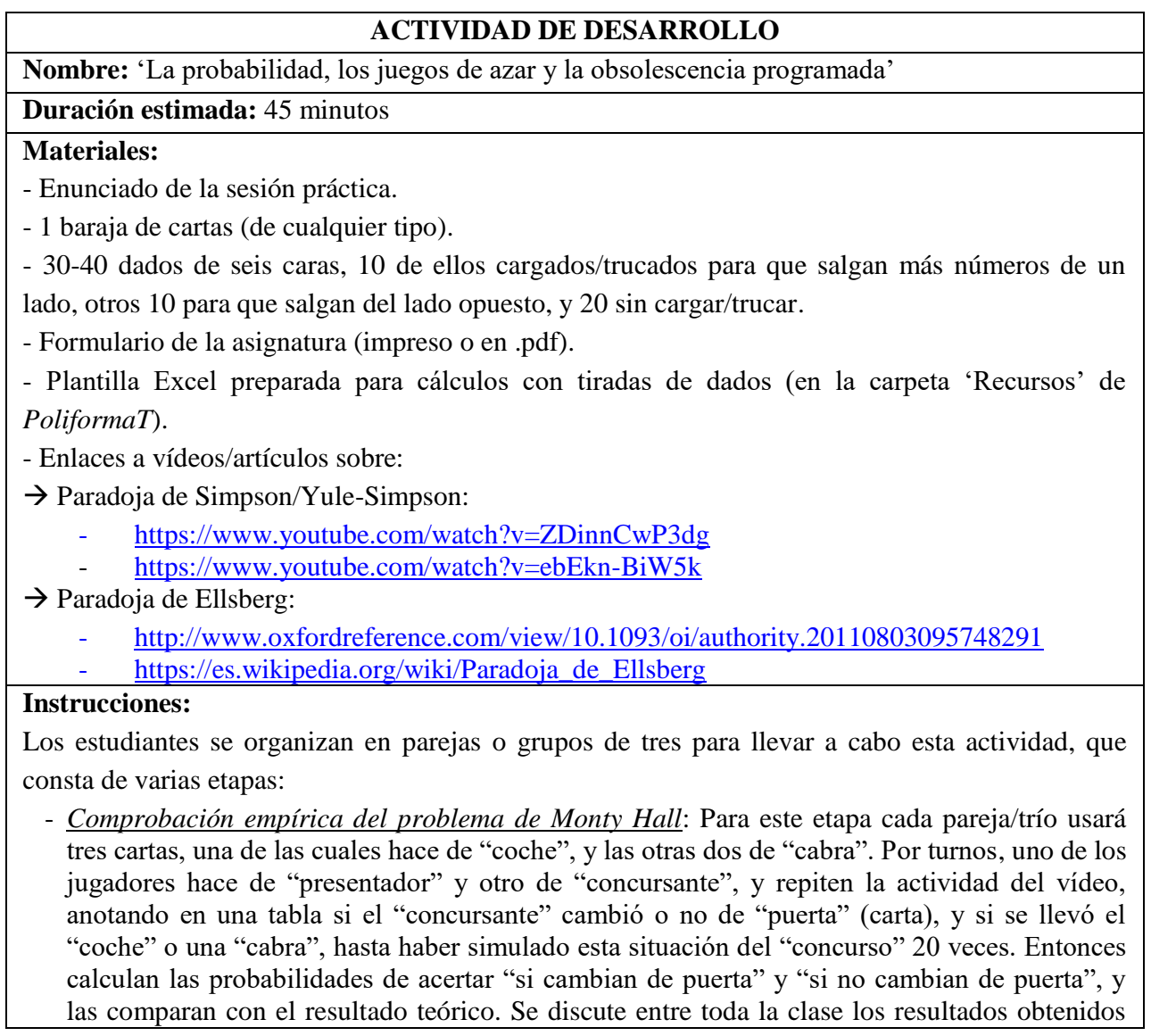




\begin{tabular}{|l|}
\hline ACTIVIDAD DE DESARROLLO \\
\hline por cada equipo, y se agrupan todos los datos para hacer un cálculo más preciso. Esta etapa no \\
debe durar más de 10 minutos en la recogida de datos, y 5 en la discusión posterior. \\
- Paradoja de Ellsberg: esta etapa se preguntará a mano alzada qué estudiantes habrían \\
cambiado o no de puerta en el caso anterior, y se les presentará un problema asociado con la \\
"paradoja de Ellsberg" y se preguntará también qué opción de dos ofrecidas escogería cada \\
uno (a mano alzada de nuevo). Luego se discute la paradoja de Ellsberg y su relación con las \\
dos preguntas planteadas. Esta etapa no debe durar más de 10 minutos. \\
- Paradoja de Simpson/Yule-Simpson: en esta etapa, cada grupo tendrá 3-4 dados. Los dados se \\
reparten, además, de manera que algunos grupos tengan dados cargados en un sentido, otros \\
sólo en un sentido, y otros no tengan ningún dado cargado. El profesor deberá saber qué dados \\
están cargados y cuáles no, pero no se proporcionará esta información a los estudiantes, a \\
priori. Los alumnos hacen 10-15 tiradas con cada dado, y anotan en el documento de Excel \\
proporcionado los resultados de las tiradas para cada uno de los dados. La plantilla hará los \\
cálculos por ellos. Es recomendable que el profesor lleve a cabo la actividad por su cuenta \\
antes de la práctica y disponga de su propia hoja con resultados con todos los dados de \\
antemano en el momento de realizar la actividad y posterior discusión, para agilizar la \\
presentación de resultados si fuera necesario. Se analizan en grupo los resultados y se explica \\
la paradoja de Simpson/Yule-Simpson. Esta etapa no debe durar más de 20 minutos. \\
\hline Evaluación: \\
- La evaluación en este caso se lleva a cabo mediante el debate tras cada ejercicio. Dado que es \\
posible que no todo el mundo quiera participar en igual medida, se recomienda o bien dar poco \\
peso, o ninguno, en la nota final a esta actividad, y usarla simplemente como seguimiento, o bien \\
"forzar" a todo el mundo a participar preguntando "a dedo" a cada grupo, eligiendo cada vez a un \\
representante, si esto no consume demasiado tiempo (es decir, si el grupo es más reducido).
\end{tabular}

Fuente: Elaboración Propia

Los resultados de aprendizaje que se pretenden evaluar con esta actividad son: a) Calcular probabilidades de sucesos condicionados y sin condicionar y b) Analizar las implicaciones prácticas de calcular correctamente probabilidades y de saber interpretarlas.

\section{Conclusiones}

En el trabajo se proponen una serie de actividades a aplicar en una unidad didáctica correspondiente a la asignatura de Estadística del grado de Ingeniería Química, ofertado por la UPV, con la finalidad de paliar deficiencias como: definir los problemas planteados, identificar y aplicar las herramientas matemáticas para su resolución y el análisis crítico de los resultados. Dichas actividades (previa, de inicio y desarrollo) se realizan antes y durante la sesión presencial, basándose tanto en material que el alumnado tiene disponible en PoliformaT como en el visionado de vídeos relacionados con ciertas paradojas estadísticas. Como futuras líneas de investigación se proponen evaluar el resultado de su implantación, tanto de manera global como desagregando por factores de tipo personal o académico del alumnado (estudios previos, situación laboral, etc.). 


\section{Agradecimientos}

Expresamos nuestro agradecimiento al Servei de Formació Permanent i Innovació Educativa así como a los Vicerrectorats d'Estudis de Grau i Política Lingüística i de Polítiques de Formació i Qualitat Educativa de la Universitat de València por la concesión del proyecto de innovación educativa UV-SFPIE_GER18-849069 que ha financiado parcialmente esta comunicación.

\section{Referencias}

Agudo Garzón, J.E.; Hernández -Linares, R.; Rico García, M.; Sánchez Santamaría, H. (2014).

Seguimiento y autoevaluación en el aula universitaria con una Tablet PC. Revista complutense de la educación 25 (2), pp. 185-210

Cabrera, L. (2015). Efectos del proceso de Bolonia en la reducción del abandono de estudios universitarios: datos para la reflexión y propuestas de mejora. Revista Fuenntes, 16, 39-62. doi:http://dx.doi.org/10.12795/revistafuentes.2015.i16.02

Casasús Estellés, T.; Ivars Escortell, A.; López Rodríguez, M. (2018). Present and Future of the ELearning in Economics Schools and Faculties. Multidisciplinary Journal for Education, Social and Technological Sciences. 5(1):44-64. doi:10.4995/muse.2018.9777

Authors (2014).

Romero, R. y L. Zúnica (2008). Métodos Estadísticos en Ingeniería. Editorial: UPV. Valencia.

Seaborn, K. y Fels, D.I. (2015). Gamification in theory and action: A survey. International Journal of Human-Computer Studies. 74: 14-31. doi: 10.1016/j.ijhcs.2014.09.006 